\title{
Compromiso miocárdico en la enfermedad de Chagas: del parásito a la respuesta inmune
}

\author{
Myocardial Involvement in Chagas disease: From Parasite to Immune Response
}

LUIS EDUARDO ECHEVERRÍA ${ }^{1,2 \oplus}$

La cardiopatía chagásica (CC) es un modelo único y fascinante de compromiso miocárdico sin muchos símiles dentro del espectro de la patología cardiovascular. La enfermedad de Chagas (EC) tiene su génesis en un proceso infeccioso agudo, básicamente zoonótico, producido por un protozoario hemoflagelado, denominado Trypanosoma cruzi (TC). Después de su fase aguda, la mayoría de las veces oligosintomática, la enfermedad se enfría misteriosamente y evoluciona a una fase quiescente y, varias décadas después de la primoinfección, se desencadena de forma enigmática y selectiva (en menos del $30 \%$ de los portadores asintomáticos) el compromiso orgánico que involucra en la mayoría de los casos al sistema cardiovascular. (1) Ya en este punto, nos enfrentamos a una de las cardiomiopatías más letales conocidas en el área de la medicina humana, que implica casi invariablemente: compromiso prototípico del sistema de conducción, una furiosa generación de arritmias supraventriculares y ventriculares acompañadas de alto riesgo de muerte súbita, insuficiencia cardíaca de acelerada evolución hacia la refractariedad y, además, como si fuera poco ya, convierte al corazón en una eficiente factoría tromboembólica. (2) Esta última característica, no solo es explicada por la frecuente presencia del paradigmático aneurisma apical y el aumento de casos de fibrilación auricular, sino también por probables factores intrínsecos de hipercoagulabilidad propios del patógeno. (3)

¿Pero cómo es posible que este microscópico protozoario produzca todo este daño? Disautonomía, alteración microvascular, fenómenos microembólicos y fibrosis focal son los mecanismos más relacionados con el compromiso cardíaco, sin embargo, realmente son solo epifenómenos que apenas nos dejan ver la superficie de la patogénesis de la enfermedad de Chagas. Los verdaderos determinantes subyacen en la sombra, ocultos por la escasez de recursos de investigación, mínimo interés en la enfermedad o simplemente por un insuficiente entendimiento de todas las variables de la biología humana. Dos de ellos son los sospechosos habituales y las líneas principales de investigación en EC. El primero está relacionado con patogenicidad directa por el parásito y el segundo, por la respuesta inmune específica a los antígenos de T. cruzi ya sea por cito- toxicidad directa celular/humoral o por autoinmunidad secundaria a mimetismo molecular, que convierten al miocardiocito en una víctima colateral de la respuesta al patógeno. (4) En resumen: parásito o inmunidad.

En este número de la Revista Argentina de Cardiología, Principato y colaboradores aportan luces sobre este tema y traen a la mesa un interesante estudio cuyo objetivo es revelar si hay un patrón inflamatorio distintivo de aquellos pacientes que presentan alteración miocárdica incipiente secundaria a CC. (5) Para este fin, los investigadores evaluaron el perfil inflamatorio de 22 pacientes con diagnóstico de enfermedad de Chagas sin deterioro de la función sistólica. Diez de ellos presentaban trastornos intraventriculares de la conducción (TIVC) y fueron contrastados con los 12 restantes sin este hallazgo. A pesar del reducido tamaño de muestra, se evidenció de forma convincente un perfil inflamatorio diferencial con un incremento estadísticamente significativo de citoquinas inflamatorias, tales como MP1 $\alpha$, las IL 2, 12, IL 15 e INF- $\gamma$ en el grupo con TIVC con respecto al comparador. Adicionalmente, el perfil inflamatorio de los individuos sin TIVC se contrasto con un grupo control sin enfermedad sin encontrar diferencias significativas. En resumen, los pacientes con estadio B1 de la CC tenían un aumento de citoquinas inflamatorias no presente en el grupo sin alteraciones de la conducción interventricular o en el grupo control.

Existen varios hallazgos que apoyan un compromiso inmunológico como un factor clave dentro de la génesis de la CC. Es interesante que, a pesar de la exposición universal al parásito, solo un tercio de los individuos infectados progresa a la fase determinada lo que sugiere que el parásito por sí mismo no es suficiente. Otro hallazgo llamativo, consiste en la descripción de agregación familiar de casos de CC, lo que sugiere un elemento de susceptibilidad genética o respuestas inmunológicas predeterminadas. (6) Es interesante también la observación de la escasa correlación entre la intensidad de la reacción inflamatoria y el número de parásitos. (7) En ese mismo sentido, recientemente, nuestro grupo de investigación describió como, de forma notablemente inesperada, individuos con CC con baja parasitemia tenían un compromiso mayor de la función sistólica, mayor alteración de la deformidad

Rev Argent CARDiol 2021;89:89-91. http://dx.doi.org/10.7775/rac.es.v89.i2.20183

VER ARTÍCULO RELACIONADO: Rev Argent Cardiol 2021;89:130-134. http://dx.doi.org/10.7775/rac.es.v89.i2.19866

\footnotetext{
${ }^{1}$ Director del programa de insuficiencia cardiaca y trasplante de corazón. Fundación Cardiovascular de Colombia

${ }^{2}$ Director del consenso científico de enfermedad de Chagas. Sociedad Interamericana de Cardiología - E-mail: luisedo10@gmail.com
} 


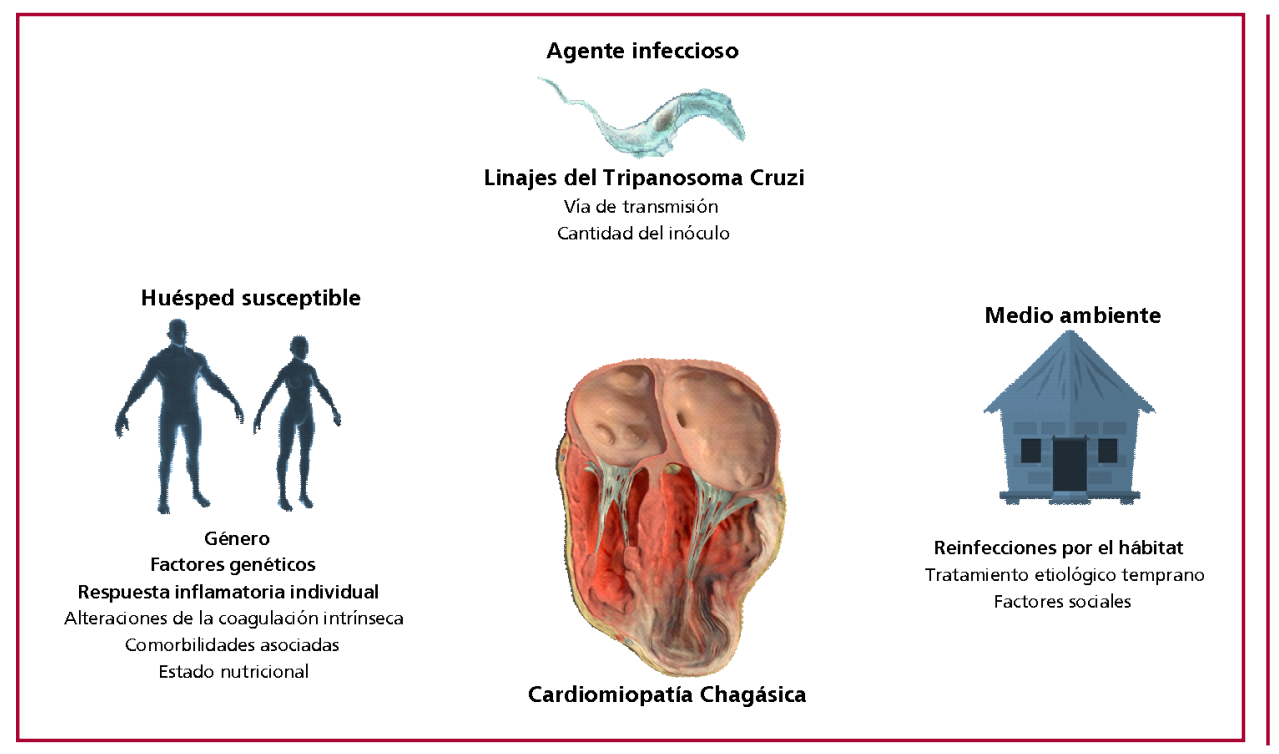

Fig. 1. Tríada ecológica de la enfermedad de Chagas, donde la interacción huésped, agente y medio ambiente crea múltiples posibilidades para desarrollos diferenciales de la enfermedad.

miocárdica medida por técnicas de strain, un aumento estadísticamente significativo de biomarcadores cardíacos y mayor mortalidad a dos años de seguimiento comparados con aquellos con parasitemia elevada. (8) Todos estos datos, apoyan la hipótesis de que el aclaramiento inmunológico del parásito podría ser aún más importante que la presencia de este en sí mismo y que, la reacción inflamatoria particular de cada organismo es potencialmente la clave en el rompecabezas del daño miocárdico en CC. Otro tópico de no menor importancia es determinar cómo esta actividad inmunológica produce daño. ¿Podría el Trypanosoma cruzi ser nuestro pariente lejano evolutivo, y conservamos algunos rasgos de familia que terminan siendo nuestra perdición? La preservación evolutiva de las secuencias primarias de muchas proteínas y sustancias estructurales de los protistas en la evolución hacia el homo sapiens apuntan en esa dirección; no es sorprendente, por lo tanto, que se pueden detectar antígenos de reacción cruzada entre las dos especies. Artículos sobre la reactividad cruzada inmunológica y mimetismo antigénico entre $T$. cruzi y autoantígenos del hospedador han sido publicados previamente. (9) Vermelho y colaboradores describieron, hace ya tres décadas, como glicoesfingofosfolípidos del músculo cardíaco eran impactantemente similares a aquellos constitutivos del $T$. cruzi y podían inducir reactividad inmunológica. (10)

Se hace patente en CC como en muchas otras enfermedades que la conjunción entre agente infeccioso, huésped y ambiente (descrita por Leavell y Clark en su legendario libro sobre medicina preventiva hace más de 50 años) (11) es crítica para el desarrollo de patología. Un ejemplo de eso recae en el mismo parásito. El linaje de T. cruzi parece determinar en gran parte el tipo de órgano comprometido ( $T$. cruzi I, compromiso cardíaco preferente; T. cruzi V y IV, compromiso cardíaco y gastrointestinal), mientras que el medio ambiente trae variables, como la posibilidad de reinfección y de adquirir comorbilidades sinérgicas. Finalmente, la respuesta innata inmunológica parece ser la clave del inicio y la extensión del daño a órganos (Figura 1).

Finalmente, y, a pesar de las limitaciones del artículo, como el pequeño número de muestra y la ausencia de correlación con otros parámetros más robustos de compromiso cardiovascular, la investigación de Principato y colaboradores aporta conocimiento clave en el entendimiento del rompecabezas que significa la $\mathrm{CC}$, sobre todo, se constituye en una valiente invitación a cardiólogos, internistas y otros profesionales del área sanitaria a explorar más allá del espectro visible de nuestro entendimiento, abandonar nuestra zona de confort y tratar de encontrar por nuestros propios medios, las respuesta a las principales preguntas de la más latinoamericana de todas las enfermedades cardiovasculares.

\section{Declaración de conflictos de intereses}

El autor declara que no posee conflicto de intereses.

(Véase formulario de conflicto de intereses de los autores en la web / Material suplementario).

\section{BIBLIOGRAFÍA}

1. Bocchi EA, Bestetti RB, Scanavacca MI, Cunha Neto E, Issa VS. Chronic Chagas heart disease management: from etiology to cardiomyopathy treatment. J Am Coll Cardiol 2017;70:1510-24. https://doi. org/10.1016/j.jacc.2017.08.004

2. Nunes MC, Beaton A, Acquatella H, Bern C, Bolger AF, Echeverría LE, et al; American Heart Association Rheumatic Fever, Endocarditis and Kawasaki Disease Committee of the Council on Cardiovascular Disease in the Young; Council on Cardiovascular and Stroke Nursing; and Stroke Council. Chagas Cardiomyopathy: An Update of Current Clinical Knowledge and Management: A Scientific Statement From the American Heart Association Circulation. 2018;138(12):e169-e209. http://dx.doi.org/10.1161/CIR.0000000000000599.

3. Echeverría LE, Rojas LZ, Gómez-Ochoa SA. Coagulation disorders in Chagas disease: A pathophysiological systematic review and meta- 
analysis. Thromb Res. 2021;201:73-83. http://dx.doi.org/10.1016/j. thromres.2021.02.025.

4. Bonney KM, Luthringer DJ, Kim SA, Garg NJ, Engman DM. Pathology and Pathogenesis of Chagas Heart Disease. Annu Rev Pathol. 2019;14:421-47.

5. Principato M, Paolucci A, Miranda S, Lombardi MG, Sosa G, Von Wulffen MA, et al. Asociación entre interleuquinas inflamatorias y la presencia de trastornos intraventriculares de la conducción en pacientes con serología positiva para enfermedad de Chagas y función ventricular conservada. Rev Argent Cardiol 2021;89:130-4. http:// dx.doi.org/10.7775/rac.es.v89.i2.19866

6. Zicker F, Smith PG, Almeida Netto JC, Oliveira RM, Zicker EMS. Physical activity, opportunity for reinfection, and sibling history of heart disease as risk factors for Chagas' cardiomyopathy. Am J Trop Med Hyg 1990;43:498-505.

7. Gironès N., Cuervo H., Fresno M. Trypanosoma cruzi-Induced
Molecular Mimicry and Chagas' Disease. Curr. Top. Microbiol. Immunol. 2005;296:89-123.

8. Echeverría LE, Rojas LZ, Rueda-Ochoa OL, Alejandro GómezOchoa S, Isabel González Rugeles C, Lucía Díaz M, Marcus R, Morillo CA. Circulating Trypanosoma cruzi Load and Major Cardiovascular Outcomes in Patients with Chronic Chagas Cardiomyopathy: A Prospective Cohort Study. Trop Med Int Health. 2020 Sep 10.

9. Kerner N, Liegeard P, Levin MJ, Hontebeyrie-Joskowicz M. Trypanosoma cruzi: Antibodies to a MAP-like protein in chronic Chagas' disease cross-react with mammalian cytoskeleton. Exp Parasitol 1991;73:451-9. 10. Vermelho AB, de Meirelles MD, Pereira MC, Pohlentz G, BarretoBergter E. Heart muscle cells share common neutral glycosphingolipids with Trypanosoma cruzi. Acta Trop 1997;64:131-43.

11. Hugh Rodman Leavell, Edwin Gurney Clark. Preventive Medicine for the Doctor in His Community: An Epidemiologic Approach. McGraw-Hill 1965. 